\title{
Estudio de los factores de riesgo psicosocial en profesoras de universidades online: Una mirada desde adentro
}

\section{Study of psychosocial risk factors in online university female teachers:}

\author{
A look from the inside \\ Mariluz García-González¹, Fermín Torrano² y Guillermo García-González ${ }^{3}$ \\ ${ }^{1}$ Doctora en Psicología de las Organizaciones. Profesora de la Universidad Internacional de La \\ Rioja, España. E.mail: marialuz.arantzazu@unir.net
${ }^{2}$ Doctor en Ciencias de la Educación. Director Académico del Máster Universitario en Preven- ción de Riesgos Laborales de la Universidad Internacional de La Rioja, España.
E.mail: fermin.torrano@unir.net \\ ${ }^{3}$ Doctor en Derecho del Trabajo y la Seguridad Social. Profesor de la Universidad Internacional \\ de La Rioja, España. E.mail: guillermo.garcia@unir.net \\ Universidad Internacional de La Rioja. \\ Logroño, España.
}

\section{Resumen}

El objetivo de esta investigación fue conocer y analizar los principales factores de riesgo psicosocial a los que están expuestas las profesoras que trabajan en universidades online. Para ello, se realizaron 20 entrevistas en profundidad fenomenológicas, tomando como referencia el modelo de Seidman, a 10 mujeres que trabajan bajo esta modalidad, para obtener una comprensión global y holística del problema a partir de sus puntos de vista y del contexto en el que se hallan inmersas. Los resultados muestran la existencia de distintos factores psicosociales de riesgo, como sobrecarga mental cuantitativa, subcarga mental cualitativa, aislamiento, conflicto de rol y sobrecarga emocional, aspectos que deberían formar parte de los programas preventivos y de intervención psicosocial adecuados a las características particulares de la mujer, lo cual contribuirá a promover una organización saludable, cuyo propósito no es solo intervenir sobre los aspectos disfuncionales, sino lograr la salud y bienestar de sus trabajadores, potenciando al máximo el desarrollo de la organización y sus empleados.

Palabras clave: Enseñanza universitaria; Profesoras online; Entrevista fenomenológica de Seidman; Sobrecarga mental; Aislamiento; Organizaciones saludables.

Abstract
The advancement and integration of new
Information and Communication Techno-
logies (ICT) in the workplace has led to the
emergence of new formulae such as telewor-
king, which have crossed the boundaries of
space and time, modifying the working stage,
the way of supervision of work and the recon-
ciliation between family and personal life.
Within this frame of reference, workers are
more exposed to new risks emerging, like the
technostress and burnout issues; as well as the
trouble reconciling professional and family


life, which result in negative effects on people, and can generate a higher level of exhaustion, dissatisfaction, and professional wear. In this context, the aim of this study is to know and analyze the main psychosocial risk factors female online university teachers are exposed. The interview on phenomenological depth, taking as reference the Seidman's model- was used as a data collection technique. In particular, two scripts, one for each of the interviews to be done, were developed and we proceeded to validate them through consultation of 15 experts from the field of safety and health and university teaching, and through the Lawshe content validity study (1975), recommended by Cardona (2006), as a quantitative validity criterion, and the observations and suggestions made by the experts as a qualitative validity criterion. In practice, 20 phenomenological interviews were conducted with 10 women working under this labor formula, to obtain a comprehensive understanding and holistic approach to the problem-from their point of view and the context in which they are immersed. The results show the existence of several psychosocial risk factors related to the position and the social environment. As regards the position-related psychosocial risk factors, the findings show the existence of quantitative mental overload, associated with intense periods of work or peaks of work, which are specific moments, linked to the dynamics of temporary teaching online and teaching in general, where work load increases substantially, doubling the amount of work to be carried out. Another psychosocial risk factor perceived negatively by participants, also refers to the qualitative mental under load, which occurs during certain academic monotonous and repetitive activities when teaching online. On the other hand, in relation to psychosocial factors associated with the social and organizational sphere, the isolation of participants working in their home is highlighted. This isolation is related both to the lack of contact with peers, to the absence of spontaneous talks and emotional support, that is, to the loss of informal interaction in the workplace, and to the need to share doubts, to work together on a common educational Project or to homogenize criteria in the same subjects taught by different teachers. On the other hand, the online female teachers reveal in their speeches different situations of personal-role conflict, in which demands are produced, focused mainly on situations of evaluation of both academic work and exams, which are incompatible with their beliefs and values. Finally, the results of the present study indicate the emotional over load due to conflicts with students, usually relating to their assessment. The above supports the need for further research in this field, aimed at developing and implementing preventive and psychosocial risk intervention programmes in the university context, which have an impact on the organizational and personal scope and which are adequate to the characteristics of women. This will help promote a healthy organization, an organization that develops healthy practices of structuring and managing work processes that would influence the development of healthy employees, whose purpose is not only to intervene on the dysfunctional aspects, but attain the welfare of its workers achieving the highest increase in the development of the organization and its employees. Keywords: Teaching university; Online female teachers; Seidman's phenomenological interviewing; Mental overload; Isolation; Welfare organizations.

\section{Introducción}

Los singulares escenarios de trabajo derivados del avance e integración de las TIC en el ámbito laboral han dado lugar a la aparición de nuevas formas de trabajo cada vez más flexibles, como el teletrabajo, que han modificado el contexto laboral, la forma de supervisión del trabajo y la conciliación de la vida familiar y profesional (Velásquez y Vera, 2018). En la actualidad, esta fórmula, definida por la Organización Internacional del Trabajo 
(2011) como el trabajo a distancia (incluido el trabajo a domicilio) efectuado con auxilio de medios de telecomunicación y/o de una computadora está en pleno auge y cuenta cada vez con una mayor presencia, especialmente, en el campo de la enseñanza universitaria online (Merino, Meneses y Ballesteros, 2008).

El teletrabajo no es ni bueno ni malo para la salud (Alonso y Cifre, 2002), sino que hay que tener en cuenta el gran número de variables que influyen en su percepción. En cuanto a los beneficios que reporta, a nivel organizacional, aumenta la productividad, reduce el absentismo, $\mathrm{y}$, en el caso de determinadas profesiones, como gestores de ventas o profesiones relacionadas con el management, la relación con los clientes se estrecha (Crawford, Maccalman y Jackson, 2011). Por otro lado, desde la perspectiva del trabajador, el teletrabajo se ha articulado como una fórmula que facilita la conciliación, a causa de la flexibilidad temporal y espacial que promueve, y el ajuste laboral a las necesidades personales y familiares.

No obstante, junto a estas potencialidades, aparecen diferentes riesgos y factores de riesgo, principalmente en el ámbito psicosocial, que es importante identificar y prevenir. Según diversos estudios previos (por ejemplo, Marshall, Michaels y Mulki, 2007), el principal factor psicosocial es el aislamiento, generado por la distancia entre el trabajador y la red social de trabajo de la organización, que puede conducir a la desmotivación y al estancamiento profesional. Otro aspecto que refieren, especialmente, las teletrabajadoras que trabajan en sus domicilios y que afrontan obligaciones familiares, es la dificultad para delimitar los espacios de cada respectiva esfera (Pérez y Gálvez, 2009). Las interrupciones, la falta de privacidad y, muchas veces, de espacio propio, dificulta la conciliación. Asimismo, Querol y Sáez (2011) señalan que un factor de riesgo muy frecuente es el de las jornadas interminables. La posibilidad de estar conectado en todo momento facilita esta conexión permanente. De hecho, en la última encuesta Eurofound e ILO (2017) se apunta la tendencia en los tele- trabajadores, bien sean a domicilio o móviles, a trabajar más horas en comparación con aquellos trabajadores que desempeñan su actividad en un entorno físico de trabajo. Ocurre, además, que el aumento de las horas de trabajo y la sobrecarga de trabajo se relaciona con la adicción al trabajo (Alonso y Cifre, 2002).

El ámbito de aplicación del teletrabajo es muy amplio, y la docencia es uno de los campos en los que más se ha extendido actualmente dando lugar a la teleformación o e-learning. Cabero (2006) señala que la teleformación tiene muchas ventajas de tipo económico: ahorra costos y desplazamientos; metodológicas: proporciona flexibilidad y autonomía en el aprendizaje; y las propiamente formativas o educativas: potencia la adquisición de habilidades y competencias necesarias para el éxito personal y la inserción profesional de los alumnos. Por otra parte, perfila las limitaciones o debilidades más importantes de la teleformación, entre las que destacan la brecha digital y la escasa formación y competencias de profesores, y también de alumnos.

En este escenario aparece un nuevo perfil profesional, el de profesor online, que varía sustancialmente del profesor tradicional a nivel pedagógico y metodológico. Desde el ámbito laboral, el desarrollo de esta nueva figura, ligada estrechamente a la dinámica y evolución de las TIC, comporta también nuevos riesgos laborales, los denominados "riesgos nuevos y emergentes" (Brocal, 2016). En este sentido, resulta prioritario identificar y profundizar en aquellos factores psicosociales de riesgo implicados en su aparición y desarrollo.

\section{Principales factores de riesgo psicosocial en profesoras online}

Tradicionalmente, la investigación en el campo de la Seguridad y Salud en el Trabajo (SST) se ha centrado prioritariamente en el estudio de los riesgos físicos e higiénicos y, prioritariamente, en los trabajos manuales habitualmente desempeñados por los hombres, infravalorándose en este sentido los 
peligros para la salud y prevención en mujeres (Valenzuela y Mora, 2009). Sin embargo, con la preponderancia que han ido adquiriendo los aspectos psicosociales en la investigación (Pulido, Puentes, Luney, López y García, 2015), esta orientación ha ido modificándose (Artazcoz, 2014).

En el estudio sobre factores psicosociales de riesgo en profesores online realizado por Baraza, Rimabu, Dalmau y Llacuna (2015) se concreta la cantidad de tiempo dedicado al teletrabajo, cuyo porcentaje ideal se sitúa entre un $20 \%$ y un $50 \%$, y se evidencia el hecho de que la doble presencia de la mujer (en la esfera familiar y profesional) mejora mínimamente con la situación de teletrabajo, ya que se solapan tareas domésticas y familiares con el trabajo. Otro trabajo relevante llevado a cabo en el marco de la Unión Europea por la European Trade Union Committee for Education durante los años 2010-2011, en el que se pone de relieve que los factores de riesgo psicosocial valorados más negativamente por los profesores tienen que ver con la sobrecarga de trabajo/trabajar con intensidad y, en segundo lugar, con la sobrecarga de rol por el desempeño de múltiples tareas. Junto a estos estudios, se destaca un proyecto desarrollado en el ámbito universitario por Domínguez (2004), que revela la existencia de estresores significativos en este contexto, asociados a la falta de reconocimiento en términos de promoción, la compatibilización de docencia e investigación, y el bajo salario percibido, especialmente, entre aquellos que están a tiempo parcial, con una elevada carga docente. Por otro lado, Castaño (2010) señala que el teletrabajo puede contribuir a invisibilizar el trabajo de la mujer y a su aislamiento, dificultando su promoción laboral, tal y como se pone de manifiesto también en el estudio realizado por la Eurofound e ILO (2017). Finalmente, una de las investigaciones que se destaca fue desarrollada por Gálvez (2008), en la que se identificaron las estrategias de conciliación utilizadas por un grupo de teletrabajadoras. Para ello, se aplicaron entrevistas en profundidad semiestructuradas a 24 mujeres y se formaron distintos grupos de discusión en los que participaron 53 personas. Las conclusiones reflejan que las empleadas necesitan del apoyo social familiar para poder conciliar y que, aunque el teletrabajo constituye una buena opción para poder conciliar, se produce una sobrecarga de trabajo, implicando además una renuncia asumida al desarrollo profesional. De modo que sigue evidenciándose que esta fórmula laboral presenta una doble dimensión, positiva y negativa.

Bajo el marco de referencia descrito anteriormente, el objetivo del presente estudio fue conocer y analizar los principales factores de riesgo psicosocial que son percibidos de manera significativa y negativa por las mujeres que trabajan como profesoras online de universidad. Este interés responde, por un lado, a la preocupación por los riesgos psicosociales y los factores organizacionales asociados que se recogen, tanto en la literatura especializada (por ejemplo, Cuervo, Orviz, Arce y Fernández-Suárez, 2018), como en los distintos ámbitos y organismos políticos encargados del desempeño saludable del trabajo (Agencia Europea para la Seguridad y Salud en el Trabajo, 2016) y, por otra parte, a la evidencia de que la evaluación de este tipo de aspectos constituye la gran asignatura pendiente de las organizaciones (Moreno-Jiménez y Garrosa, 2013). Junto a lo anterior, este trabajo se incardina en la concepción preventiva que persigue el bienestar laboral dispuesta por la Directiva Europea Marco 89/391/CEE, relativa a la aplicación de medidas de promoción de la seguridad y salud de los trabajadores en el lugar de trabajo, transpuesta en el contexto legal español por la Ley 31/1995, de prevención de riesgos laborales.

\section{Método}

\section{Técnica de datos}

Se empleó la entrevista en profundidad fenomenológica, para obtener una compren- 
sión global y holística del problema a partir de los puntos de vista de las profesoras online y del contexto en el que se hallan inmersas (Riba, 2007). En concreto, se tomó como referencia el modelo de entrevista en profundidad fenomenológica de Seidman (2006), adecuada para investigadores que están interesados en las historias de otros, en comprender la experiencia de esos otros y los significados que les dan. Las entrevistas fueron individuales, semiestructuradas y en formato online, y se combinaron respuestas abiertas, que dejaban un amplio margen para la improvisación, con respuestas más cerradas, que se pospusieron para el final de la entrevista o cuando se consideró necesario profundizar o abordar algún aspecto concreto.

En la primera toma de contacto con las participantes se les explicó el propósito del trabajo de investigación y cómo se desarrollarían ambas entrevistas, así como el archivo confidencial de la información y su uso, preservando en todo momento el anonimato de las participantes. Una vez obtenida la confirmación de su participación desinteresada en la investigación, se les remitió un segundo e-mail, en el que se adjuntó el documento de consentimiento informado para que lo revisaran y, en caso de estar de acuerdo, lo devolvieran firmado. En la elaboración del consentimiento informado se siguieron y respetaron las recomendaciones de la UK Data Archive.

\section{Procedimiento de diseño y validación de los guiones de entrevista}

El proceso de validación se desarrolló en dos fases claramente diferenciadas: el diseño del guion y la consulta a expertos. En relación con la primera, se elaboraron dos guiones de entrevistas (ver Cuadros 1 y 2), en función de una revisión de la literatura exhaustiva de acuerdo con las pautas y orientaciones para trabajar documentación en investigación cualitativa. En concreto, se siguieron las recomendaciones de Lichtman (2013), en las que a partir de una búsqueda amplia de información y su posterior análisis en función de su relevancia, se ordena y organiza dicha información de modo narrativo conformando un modelo conceptual. A partir de este modelo conceptual se articularon los objetivos y preguntas de investigación que orientaron el diseño y construcción de los dos guiones.

La primera entrevista hace referencia a la historia pasada de la profesora online y se centra en el abordaje de aquellos aspectos, cuya exploración biográfica pasada aporta información necesaria y relevante para comprender su historia presente. Se redactaron para esta primera entrevista 12 preguntas en consonancia con los objetivos de investigación correspondientes. El segundo guion de la entrevista está formado por 40 preguntas $\mathrm{y}$, al igual que el primero, se formularon en coherencia con los objetivos del estudio, si bien se centran en la historia de vida presente de las docentes.

\section{Cuadro 1}

Guion validado de Entrevista 1.

1. ¿Podría describir cómo ha sido su trayectoria formativa, desde sus inicios escolares hasta la actualidad?

2. ¿Cómo ha sido su relación con las nuevas tecnologías desde la primera toma de contacto hasta hoy, tanto en el ámbito laboral como fuera de él?

3. Hasta el momento ¿podría detallar las facilidades y dificultades que ha encontrado para conciliar su vida personal y su trabajo?

4. A lo largo de su trayectoria profesional, ¿Qué papel han jugado sus familiares y amigos en relación a la conciliación entre obligaciones familiares y laborales? 
5. ¿Qué situaciones adversas ha tenido que enfrentar a lo largo de su vida laboral y personal? ¿Qué hizo?

6. Y ¿Qué retos ha tenido que enfrentar a lo largo de su trayectoria profesional y su vida personal? ¿De qué manera lo hizo?

7. ¿Qué problemas de salud ha tenido a lo largo de su vida hasta el momento actual?

8. ¿Podría describir con qué factores considera que están relacionados estos problemas de salud?

9. ¿Podría describir cuáles han sido sus hábitos de vida saludables y no saludables hasta el momento actual?

10. ¿Podría describir cuál ha sido su trayectoria profesional hasta el momento actual?

11. ¿Cómo valora estas experiencias laborales previas?

12. ¿Qué significado ha tenido el trabajo a lo largo de su vida?

\section{Cuadro 2}

\section{Guion validado de Entrevista 2.}

1. ¿Podría explicarnos en qué consiste su trabajo actual?

2. ¿Podría describir y valorar las características de las tareas que desarrolla en su trabajo como profesora online?

3. ¿Cómo describe y califica su ritmo de trabajo?

4. ¿Podría explicar qué se espera de usted como profesora online en el ámbito de su empresa?

5. En su trabajo, ¿cuáles son las exigencias laborales que percibe incompatibles y le generan conflicto?

6. ¿Cómo son las relaciones con sus compañeros y con sus supervisores?

7. ¿De qué manera cree que puede influir en las relaciones el hecho de realizar el trabajo a distancia?

8. ¿Cuáles son las exigencias a las que tiene que responder en la relación con sus alumnos, compañeros de trabajo y supervisores?

9. ¿Cuál es su nivel de formación y cuáles sus habilidades para el uso y manejo de las TIC? ¿Cómo los valora?

10. Según su experiencia profesional, y desde el punto de vista personal y profesional, ¿cuáles son las ventajas e inconvenientes del uso de las TIC en su trabajo?

11. ¿Podría describir cómo se desarrolla un día habitual de su vida, desde que se levanta hasta que se acuesta?

12. En su caso, ¿cuáles son los factores y características familiares y laborales que dificultan y facilitan la conciliación trabajo-familia?

13. ¿Cuáles son sus condiciones de trabajo actuales, en cuanto al tipo de contrato, remuneración y permanencia? ¿Cómo las valora?

14. ¿Podría describir cómo ha sido la evolución de su empresa, desde que usted trabaja en ella, y cómo se ha reflejado en los trabajadores?

15. ¿Podría describir los objetivos de su empresa, y en su caso departamento, y sus características?

16. ¿Podría explicar cómo es la comunicación con sus supervisores y con sus compañeros de trabajo?

17. ¿Podría describir y evaluar las características de su espacio de trabajo y de los equipos que utiliza? 
18. ¿Qué mecanismos de participación activa de los trabajadores (expresión de opiniones, participar en planteamiento de objetivos etc.) existen en su empresa?

19. ¿En su empresa y en sus tareas, de qué manera se toman las decisiones respecto a qué hacer, cómo hacerlo y, en qué plazos de tiempo?

20. ¿Podría explicar de qué manera evalúan su trabajo? ¿Cómo conoce el resultado de su evaluación?

21. Si solicita apoyo ante problemas técnicos o dificultades laborales ¿Con qué recursos y personas de su trabajo cuenta para ayudarla?

22. ¿Podría describir los criterios y posibilidades de promoción de su centro de trabajo?

23. ¿Qué medidas para favorecer la conciliación familiar oferta su empresa?

24. ¿Cuáles son las facilidades y/o ofertas formativas que le ofrece su centro de trabajo para actualizar sus conocimientos y destrezas laborales?

25. ¿Podría describir cuál cree que es su grado de competencia para poder desempeñar su trabajo de manera eficaz?

26. ¿Qué grado de competencia cree que tiene para identificar distintas maneras de alcanzar sus propósitos en el trabajo y elegir aquella que le llevará a conseguirlo?

27. ¿Podría hablar sobre su grado de motivación para lograr sus propósitos en el trabajo?

28. ¿De qué factores cree que dependen sus éxitos laborales y de cuáles sus fracasos?

29. ¿En situaciones laborales de incertidumbre, piensa/espera lo mejor o lo peor?

30. Piense en situaciones laborales difíciles que haya superado ¿que consecuencias extrae de estas experiencias?

31. ¿En la actualidad, podría explicar que situaciones adversas laborales tiene que afrontar en su trabajo?

32. ¿Qué piensa y hace para enfrentarlas?

33. ¿Qué situaciones críticas considera será necesario afrontar en un futuro próximo o a medio plazo? ¿Qué hace para prevenirlos?

34. Y ¿retos?

35. ¿Qué piensa y hace para enfrentarlos?

36. ¿Qué retos considera será necesario afrontar en un futuro próximo o a medio plazo? ¿Se considera capaz de llevarlos a cabo?

37. ¿Qué piensa y siente cuando tiene que ir a trabajar? ¿Y en el trabajo? ¿Y cuando finaliza?

38. ¿Qué pensamientos y emociones suscita en usted cualquier referencia a su trabajo?

39. ¿Qué significado tiene el trabajo como profesora online para usted actualmente?

40. ¿Podría realizar una comparación en forma de metáfora, que refleje su trabajo como profesora online?

En la segunda fase se procedió a realizar la consulta a expertos. En este caso se seleccionaron 15 especialistas en el ámbito de la prevención de riesgos laborales y la enseñanza superior, para valorar la idoneidad, utilidad y pertinencia de los ítem. Asimismo, se utilizó el índice de validez de contenido de Lawshe (1975), recomendado por Cardona (2006), como criterio de validez cuantitativo, y las observaciones y sugerencias realizadas por los expertos como criterio de validez cualitativo. Como se puede ver en la Tabla 1, en 
ambos casos el índice de validez está próximo a la unidad (.97 y .98 , respectivamente), por lo que, tomando como referencia el estudio de Lawshe (1975), en el que se establece que un índice de .51 es suficiente y estadísticamente significativo con un panel de 14 expertos, se considera que la validez de contenidos de ambos guiones es la adecuada.

Tabla 1

Índice de Validez de Contenido de Lawshe (1975).

\begin{tabular}{c|cc} 
& Guion de entrevista 1 & Guion de entrevista 2 \\
\hline $\begin{array}{c}\text { Índice de Validez de } \\
\text { Contenido }\end{array}$ & .97 & .98
\end{tabular}

\section{Muestra: Selección de las participantes}

Se decidió realizar un muestreo teórico a priori, en el que se consideraron, como características importantes que permitieron homogeneizar la muestra, el género y el desempeño de la actividad laboral de docencia online fuera de su centro de trabajo. Se consideró, además, la complejidad y diferentes perspectivas de los factores de riesgo y riesgos psicosociales de naturaleza subjetiva y, por ello, se determinaron otras características que representaban esta complejidad: la existencia o ausencia de cargas familiares y el carácter exclusivo o complementario del trabajo en teleformación.

Como técnica para seleccionar los participantes, se empleó el muestreo de bola de nieve o multiplicador, que consiste en localizar informantes, pidiendo a otras personas que identifiquen a individuos o grupos con un conocimiento especial del fenómeno (Ulin, Robinson y Tolley, 2006). Se contactó, en este sentido, con compañeros investigadores, que refirieron informantes pertinentes, quienes a su vez, recomendaron otros informantes, de acuerdo con perfil delimitado.

Como se observa en la tabla 2, la muestra productora está formada por diez profesoras online, entre 34 y 59 años, de diferentes comunidades españolas, a las que se le realizaron dos entrevistas fenomenológicas en profundidad. Siete de ellas desarrollan su actividad en el domicilio familiar y las otras tres desempeñan parte de su labor en el domicilio (una de ellas también en su centro de trabajo). Respecto de las cargas familiares, seis tienen responsabilidades familiares y cuatro no las tienen. De las primeras, cuatro cuentan con niños menores de edad, una quinta comparte el domicilio y cuida de su padre, y la última de ellas está al cargo de su madre y de niños menores de edad. En relación con la exclusividad del trabajo, la mayor parte de la muestra, concretamente ocho de las informantes, desempeñan como actividad principal la teleformación; en el caso de las otras dos participantes constituye una actividad complementaria a su trabajo principal. Por último, seis de las profesoras trabajan en exclusiva en una universidad privada, dos en una escuela de grado universitario privada también, y las dos restantes desempeñan su actividad laboral de teleformación en dos universidades, una pública y otra privada, de manera simultánea.

Tabla 2

Perfil de las participantes.

Participante Edad Cargas Familiares

$\begin{array}{ccc}\text { Elena } & 51 & \text { Dos hijos edad escolar } \\ \text { Daniela } & 42 & \text { Sin cargas }\end{array}$

Actividad Prof.

$\begin{array}{cc}\text { Coordinadora / } & \text { Empresa privada/Universidad } \\ \text { profesora } \text { online } & \text { privadaública } \\ \text { Gestora / Profesora } & \text { Empresa privada/Universidad } \\ \text { online } & \text { privada/pública }\end{array}$




\begin{tabular}{|c|c|c|c|c|}
\hline Participante & Edad & Cargas Familiares & Actividad Prof. & Organización \\
\hline Laura & 38 & $\begin{array}{l}\text { Un niño edad pre- } \\
\text { escolar }\end{array}$ & Profesora online & Universidad privada \\
\hline Marta & 39 & $\begin{array}{l}\text { Un niño edad pre- } \\
\text { escolar }\end{array}$ & Profesora online & Escuela privada \\
\hline Paqui & 43 & Padre & Profesora online & Universidad privada \\
\hline Rosa & 46 & $\begin{array}{c}\text { Dos hijos edad escolar / } \\
\text { Madre }\end{array}$ & $\begin{array}{l}\text { Profesora online / } \\
\text { Coordinadora }\end{array}$ & Universidad privada \\
\hline Clara & 58 & Sin cargas & Profesora online & Escuela privada \\
\hline Patricia & 34 & $\begin{array}{l}\text { Un niño edad pre- } \\
\text { escolar }\end{array}$ & Profesora online & Universidad privada \\
\hline Julia & 34 & Sin cargas & Profesora online & Universidad privada \\
\hline María & 59 & Sin cargas & Profesora online & Universidad privada \\
\hline
\end{tabular}

\section{Análisis de los datos}

El análisis de la información recogida en las entrevistas se realizó a través de dos ciclos analíticos: transcripción de su contenido y segmentación y codificación. En concreto, las entrevistas se grabaron en formato audiovisual y online, recogiendo el contenido verbal y aquellos aspectos de la comunicación no verbal más relevantes. Se utilizó para la transcripción el programa Maxqda. La transcripción de cada entrevista quedó vinculada con su registro audiovisual, lo cual ofrece la posibilidad de revisión y relectura simultánea. Durante la transcripción de la entrevista, de acuerdo con los criterios éticos en investigación, se preservó en todo momento el anonimato de las participantes. Del mismo modo, se emplearon pseudónimos en todos aquellos datos que facilitaron la identificación de las participantes tales, como lugares, escuelas, organizaciones y nombres de personas relacionadas con ellas y mencionadas durante la entrevista (Seidman, 2006).

Con los datos cualitativos que se generaron

en las entrevistas, se realizaron dos lecturas: una primera lectura, para aprehender el significado global de la entrevista, y una segunda lectura, en la que se segmentó en unidades significativas más pequeñas y manejables que se convirtieron en contextos de la codificación. Asimismo, el proceso de codificación se realizó en dos fases. En el primer ciclo de codificación se elaboró una lista de códigos inicial a priori de acuerdo con los objetivos y preguntas de investigación y, en la segunda fase, se codificó la información por patrones.

\section{Resultados}

En la figura 1 se puede observar que, entre los factores psicosociales de riesgo asociados al puesto, emerge con mayor fuerza del discurso de las participantes la sobrecarga mental cuantitativa, estrechamente vinculada a los picos o crestas de trabajo. Además, asociada a un tipo de tarea concreta y puntual, como es la corrección de actividades y trabajos académicos, se produce una condición de subcarga mental cualitativa. 


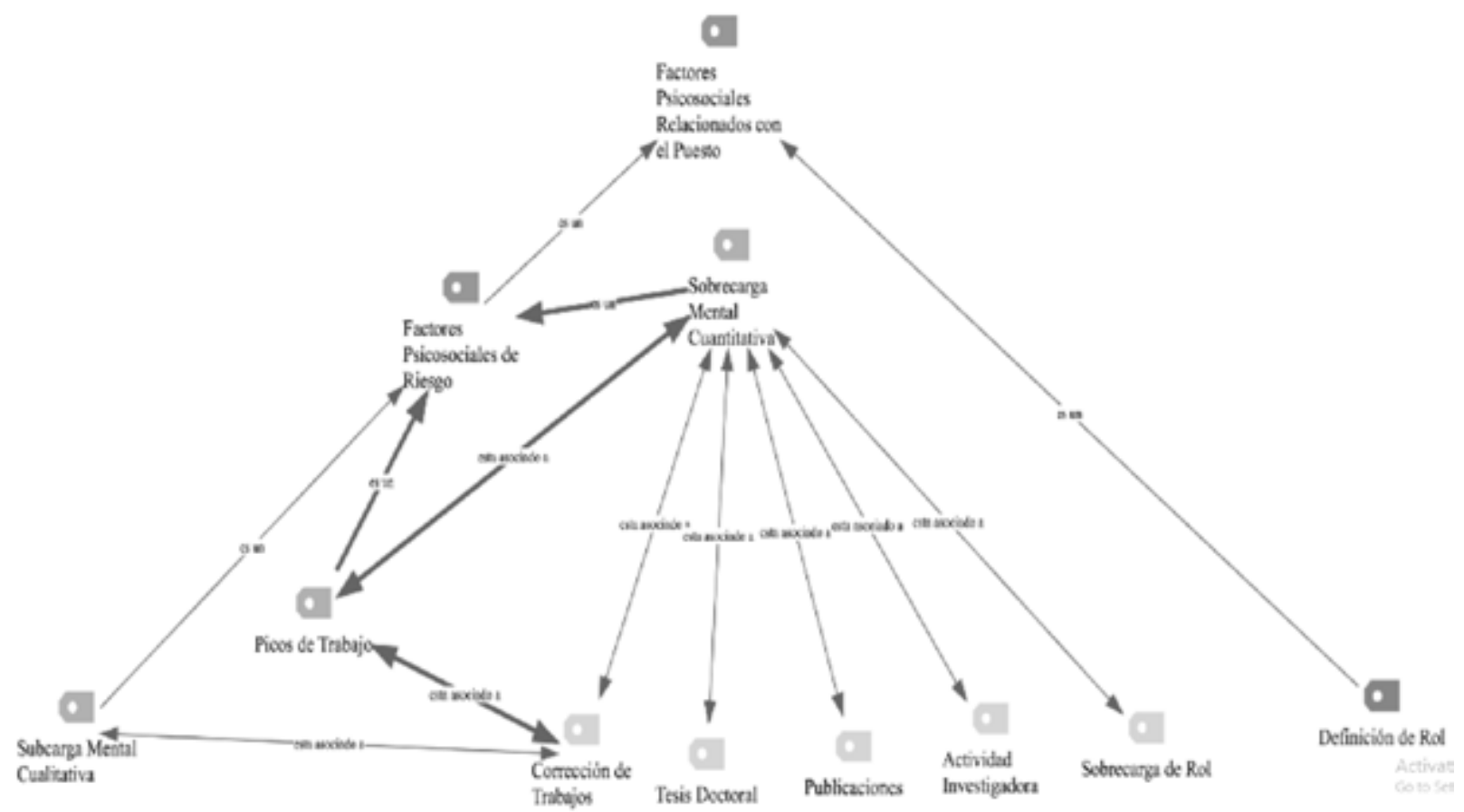

Figura 1. Factores psicosociales de riesgo relacionados con el puesto.

Las profesoras online refieren en sus narraciones que la naturaleza de las tareas que realizan es básicamente cognitiva, precisando una importante exigencia de atención, memoria de trabajo y razonamiento, aspectos característicos de la fatiga mental (Sebastián y Del Hoyo, 2002):

"El que trabaja en un trabajo manual pues se le carga la espalda o se le cargan las piernas, pues bueno, creo que la carga nuestra es más de la cabeza, más cognitiva" (Elena, 1.2-12:9).

Esta carga mental se refleja en las distintas tareas docentes que deben desempeñar, unas ligadas al rol tradicional de profesor y otras más específicas de la modalidad online. Respecto de las primeras, destacan el diseño y planificación de actividades, la preparación de exámenes y la corrección de trabajos y pruebas de evaluación. Las tareas derivadas de la enseñanza online se materializan en actividades de seguimiento y motivación (foros de debate, prioritariamente), preparación e impartición de clases por videoconferencia y la gestión del correo electrónico y de los foros y chats para contestar dudas. En momentos puntuales, y ligados a la dinámica temporal de este tipo de enseñanza, se producen períodos muy intensos de trabajo, a los que se refieren las participantes como "crestas" o "picos", en los que el volumen de trabajo se incrementa vertiginosamente, hasta incluso duplicar la carga laboral. Esta sobrecarga no se correlaciona con un aumento del tiempo requerido para afrontarla, sino que se debe llevar a cabo de acuerdo con los plazos establecidos en la temporalización inicial y que, frecuentemente, no se ajustan a las necesidades reales. En la siguiente cita se refleja la capacidad humana limitada de la profesora ante esta situación, frente a la de máquina que corrige sin descanso:

"Yo no soy una máquina, soy una persona, y entonces tengo que leer uno y hay que calificarlo, hay que ponerle todas las cosas que tiene que modificar, hay que quedar con el alumno" (Paqui, 5.1-66:8).

En relación con lo anterior, una de las variables que subrayan con frecuencia las docentes como origen de este desajuste volumen/ tiempo, provocando una sobrecarga cuantitativa, es el cálculo inadecuado de su carga de 
trabajo, que no responde a los requerimientos y tiempos reales ni a la jornada contractual:

"Yo, con un contrato de media jornada, tengo 120 alumnos y doce trabajos fin de máster, lo que me parece una brutalidad" (Paqui, 5.266:13).

"Se destinaba una hora a la preparación de una clase presencial, pues, obviamente, en una hora es imposible" (Julia, 9.2-28:4).

Estos picos de trabajo se generan principalmente en la entrega por parte de los estudiantes de los trabajos de actividad continuada o de final de curso, quienes comparten la tendencia a realizar la entrega el último día del plazo. El requerimiento de estas tareas, definido por una gran cantidad de trabajo que demanda una corrección rigurosa y detallada, y una valoración cuantitativa y cualitativa personalizada para cada alumno, se convierte en una exigencia personal para las participantes, que eligen aumentar su horario de trabajo, a pesar del coste familiar o personal que conlleva, en consonancia con su ética e implicación laboral:

"Lo noto porque requiere mucha atención, como te comenté, a mí, cuando un alumno me envía una actividad, no me gusta prorratear por encima, me gusta mirarla línea por línea" (María, 10.2-179:1).

La sobrecarga cuantitativa asociada a los picos de trabajo afecta a las participantes de manera negativa, generando agobio, desmotivación y dificultando la conciliación familiar. En el caso de las profesoras online con hijos en edad escolar, quienes necesitan ayuda $\mathrm{y}$ supervisión para realizar los deberes o las actividades extraescolares, estos picos de trabajo suponen una sobrecarga de rol derivada de las exigencias de ambas esferas. De modo que, para poder dar salida a este volumen de tareas, trabajan de noche o los fines de semana:

"Con el café y la Coca-Cola, porque me lo quito de horas de sueño, es que no hay otra. $\mathrm{Si}$ yo sé que tengo que corregir trabajos, o creo que tengo una carga muy elevada de correo electrónico, que correos se tienen, o que al día siguiente no podré gestionar, pues por la noche. Mi experiencia siempre es la misma" (Elena, 1.1-26:1).

Las repercusiones sobre la salud de las docentes aumentan exponencialmente en el caso de las dos participantes que desarrollan su actividad principal en una empresa privada $\mathrm{y}$, con carácter complementario, una actividad de teleformación en distintas universidades. La jornada laboral se incrementa notablemente, llegando a trabajar una media de más de 12 horas la mayor parte de los días, de modo que la sobrecarga mental cuantitativa asociada a los picos de trabajo se ve incrementada con la carga laboral derivada del trabajo principal, vivenciándose como situaciones muy estresantes, que han aprendido a normalizar. En este sentido, frecuentemente se encuentran en una situación de activación y alerta que afecta a su calidad y cantidad de sueño y, al parecer, sufren periódicamente episodios de ansiedad y depresión en uno de los casos y problemas de salud física graves en el otro, derivados de esta sobrecarga cuantitativa por sobrecarga de rol:

“Tengo muchísimo estrés, incluso con alguna predepresión, por estrés, trabajar muchísimas horas eso sí, pero bueno tampoco. Forma parte del juego" (Elena, 1.1-55:25).

La sobrecarga cuantitativa ocupa un lugar destacado como factor psicosocial de riesgo en aquellas docentes que desempeñan su actividad principal en el ámbito universitario. El rol de profesora online en estas organizaciones conlleva el desempeño de tres tipos de actividades: actividad docente, tareas de gestión y actividad investigadora. En este sentido, unida a la sobrecarga derivada de los picos de trabajo en el desempeño de la actividad docente, se encuentra la sobrecarga mental cuantitativa fruto de las exigencias en las otras actividades laborales. Una parte de las participantes que se encuentran en esta situación se halla en pleno proceso de elaboración de la tesis doctoral y en algún caso, finalizándola. Para las primeras, implica desplazamientos a centros de investigación en otras ciudades para realizar la recogida de datos en un labo- 
ratorio, que suman aproximadamente seis o siete horas más a su jornada laboral habitual:

"Sobre las siete y media o las ocho, depende del día, me voy a otro pueblo que es donde estoy haciendo el doctorado en un centro de investigación. Paso a hacer 3 horas de laboratorio y suelo llegar a casa, pues depende del día. Hoy, por ejemplo, he llegado a las 3.30, otros días llego a las 3 más o menos y bueno luego, después de comer, me suelo poner al ordenador, para trabajar ya en la parte de la empresa de la universidad, que es la empresa en la que trabajo" (Julia, 3.2-2:1).

La obtención de la tesis doctoral es un requisito necesario para poder continuar en la universidad y un paso previo en el proceso de acreditación y promoción del profesorado universitario. Estas son dos exigencias que se recuerdan continuamente en los congresos y a través de correos electrónicos, en los que les informan sobre su posición respecto a la obtención de la acreditación. Subrayan, no obstante, que no es una demanda expresa como tal y que no van a perder su trabajo, pero que en el fondo es un requerimiento obligatorio que conlleva una cierta carga de amenaza:

"Toda la plantilla son doctores, que están contratando doctores, que vas a congresos y solo hay doctores y entonces, claro, es un reto y a la vez es una amenaza porque pienso, bueno, no me echarán, pero oye, te están diciendo 'oye hay que formarse, hay que promocionar"” (Laura, 3.1-86:5).

Por otro lado, la subcarga cualitativa es un factor psicosocial de riesgo que se da de manera puntual ante ciertas actividades monótonas y repetitivas que tienen lugar en la docencia online: la corrección de trabajos de evaluación continua y la propia estructura de las actividades didácticas. La supervisión de trabajos de actividad continua, incluso en los picos o crestas laborales, se valora como una tarea especialmente rutinaria debido a que, en su mayor parte, se recogen las mismas ideas y similar exposición por la propia estructura que marca la actividad. De modo que estas características convierten esta tarea en monó- tona, tediosa y desmotivante, que requiere un importante esfuerzo por parte de la profesora online para mantener la atención:

"Pues corregir actividades, cuando tienes cien actividades que corregir en una semana la misma actividad, una y otra vez, eso la verdad que es un poco desmotivante, porque es el mismo trabajo de manera continua y continua" (Julia, 9.2-79:8).

En relación con la propia estructura de la docencia online, en la que las actividades didácticas que se configuran siguen una estructura y dinámica muy concretas, tales como foros de debate o casos prácticos que varían en contenido, pero no en forma y funcionamiento, las participantes enfatizan su carácter rutinario y repetitivo:

"Yo creo que sí, que es bastante rutinaria la formación virtual" (Elena, 1.2-76:6).

Además, indican que esta previsibilidad se produce incluso en las preguntas que realizan los alumnos, lo que permite tener ya preparadas plantillas de respuestas:

"Un $90 \%$ de lo que te preguntan o de la exposición que te plantean a nivel general del máster más o menos son siempre muy parecidas, siempre hay nuevas, pero es muy... más o menos es muy parecido lo que te preguntan de un año a otro" (Elena, 1.2-16:4).

Por otra parte, para otras participantes, las tareas que se plantean siempre pueden ser distintas, como diferentes son los alumnos, y resultar estimulantes, lo que también depende en parte de la capacidad de innovación que permita la organización en la que se trabaja. En la cita de la siguiente participante se constata esta perspectiva positiva:

"No se te puede hacer monótono, porque cada persona es un mundo y aunque después hayas visto alguna pregunta que se repite, siempre hay una nota que también le puedes encontrar, explicar una cosa distinta que hayas explicado otra vez" (Clara, 7.2-48:2).

Se evidencian, respecto a la subcarga mental cualitativa, dos tendencias marcadas, una más positiva, que se centra en los aspectos diferenciales y motivantes de las actividades 
didácticas, y una segunda negativa, que destaca ligeramente y que focaliza su atención en los aspectos más repetitivos y monótonos de las actividades y modalidad docente.

Por otra parte, en la revisión de la literatura se articuló la ambigüedad de rol como uno de los posibles factores de riesgo en la profesión de profesora online. Sin embargo, la realidad que narran las docentes se caracteriza por una definición de rol precisa. La docencia online, como se ha venido recogiendo en sus discursos, es una modalidad muy estructurada, con plazos temporales y actividades muy marcadas y, siguiendo con la misma dinámica, el rol de la persona focal está recogido de forma concisa. A este respecto, se observan dos flujos en las narraciones de las entrevistadas. Por un lado, se encuentran aquellas que reciben por escrito al inicio de cada curso un documento en el que se recogen los principales aspectos que configuran su rol: objetivos, tareas a desarrollar, medios, temporalización, normas que regulan la interacción con los alumnos y evaluación. Conocer lo que se espera de ellas proporciona seguridad a la hora de tener que enfrentarse a situaciones más conflictivas y hace que el desempeño laboral sea más fluido y ágil:

"Siempre te suelen enviar, no sé si es un pdf, pero un pdf en el que se lista qué es lo que tienes que hacer como profesor... yo tengo bastante claro qué es lo que tengo que hacer y qué es lo que se espera de mí como profesora" (Patricia, 8.2-56:6).

Por otro lado, un grupo más pequeño de profesoras online se reúnen con la coordinadora académica y todo el profesorado durante un fin de semana, para trabajar de manera constructiva sobre el proyecto educativo común. Es en este momento cuando se clari- fican los objetivos, el modelo de trabajo, las normas de actuación, etc.

"En las jornadas docentes lo hablamos, cada año, y queda muy claro. La verdad es que, antes, cuando estábamos un compañero y yo solos empezando el campus online, también lo teníamos claro, éramos dos y también. Quiero decir, ahora somos, no sé, ¿60 profesores? o 50 creo, antes éramos dos y también lo sabíamos, no sé" (Marta, 4.2-54:1).

Este aspecto es muy positivo, porque junto al planteamiento metodológico existe un planteamiento pedagógico que lo sustenta y da coherencia y sentido al proyecto educativo común. Además, proporciona a las trabajadoras la posibilidad de trabajar en equipo y de sentirse parte de la organización y de la cultura organizacional, aspecto de difícil consecución por trabajar fuera del centro de trabajo. Favorece, también, la creación de relaciones con compañeros de trabajo, que pueden funcionar como soporte académico a lo largo del curso. De manera que esta participación activa en el proyecto constituye un recurso laboral importante que satisface las necesidades básicas de pertenencia y autoeficacia, lo cual redunda en un aumento de la motivación intrínseca. Asimismo, aumenta la motivación extrínseca, porque los entornos con recursos refuerzan la dedicación al trabajo y más fácilmente se logran los objetivos (Bakker, Rodríguez-Muñoz y Derks, 2012).

Por otro lado, en cuanto a las principales demandas asociadas a la esfera sociolaboral, en la figura 2 se recogen aquellos factores psicosociales de riesgo que refieren las profesoras online en su discurso y aquellos otros aspectos que son percibidos positivamente dentro del ámbito organizacional. 


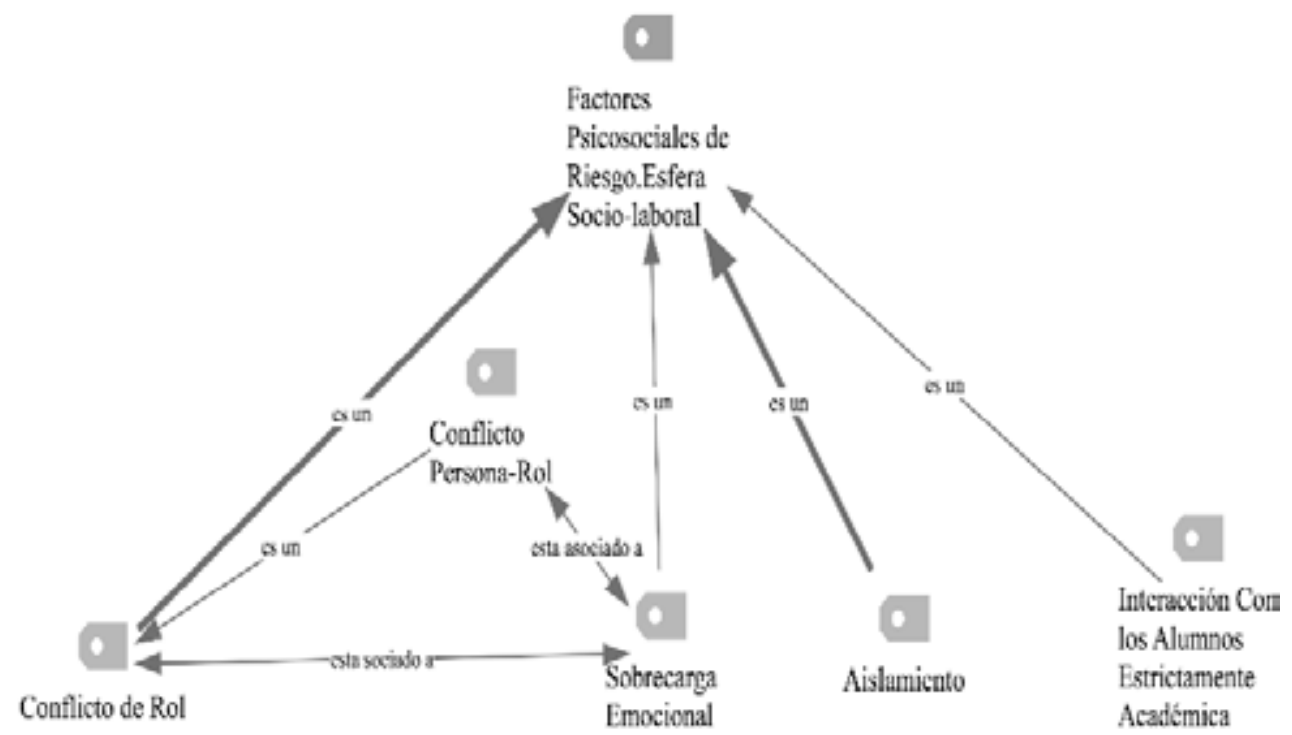

Figura 2. Factores psicosociales de riesgo relacionados con la esfera sociolaboral.

De acuerdo con los discursos de las docentes, se evidencian cuatro importantes factores de riesgo psicosocial dentro de este ámbito, entre los que destaca principalmente la sensación de aislamiento, el conflicto de rol y la sobrecarga emocional; estos dos últimos están intensamente asociados, y el aislamiento es el factor con más peso. La interacción con los alumnos estrictamente académica parece no ser valorada tan negativamente como los otros tres factores y no estar tan relacionada con la satisfacción en el trabajo.

En relación con el aislamiento, se observan en las narraciones dos tendencias marcadas que parecen estar relacionadas con el lugar donde desarrollan la actividad laboral: el domicilio o el centro de trabajo. En la primera situación, la profesora desempeña su labor aislada físicamente, mientras que en la segunda comparte espacio (despacho) con otros compañeros teleformadores.

Las participantes que trabajan en su domicilio perciben el aislamiento como el mayor factor de riesgo del trabajo online:

"Por eso, porque esa parte digamos que nos falta, el ver a la gente, el hablar. Claro, esa es la parte, desde mi punto de vista, más negativa de este trabajo: la soledad" (Rosa, 6.1-30:1).

De acuerdo con sus discursos, la principal carencia se produce en la dimensión social del aislamiento (Marshall et al. 2007), informando de la privación de contacto con sus compañeros, de la falta de charlas espontáneas y de apoyo emocional, es decir, de la ausencia de interacción informal en el ámbito laboral:

“A ver, no sé, en cierto modo es cómodo, ¿no? Estás en tu casa, pero, es verdad, que falta comunicación personal, ¿me entiendes? Eso de ir a una oficina donde tienes compañeros, yo lo echo de menos" (María, 10. 2-83:1).

Este aspecto dificulta la comunicación entre empleadas para resolver problemas del día a día, sin necesidad de acudir a los canales formales de comunicación. Entroncado también con este aspecto, señalan, haciendo alusión a la dimensión organizacional del aislamiento (Marshall et al. 2007), la necesidad de compartir dudas, de trabajar conjuntamente en un proyecto educativo común, o de homogeneizar criterios en las mismas asignaturas que son impartidas por distintos profesores. Sin embargo, la posibilidad de dar respuesta a este requerimiento no parece posible, por la falta de contacto tú a tú y por los diferentes horarios laborales y personales de cada docente que no permiten la conexión inmediata. El correo electrónico, que constituye la principal vía de contacto con los demás compañeros, implica un desgaste importante de tiempo que finalmente no resulta rentable. Cuando las entre- 
vistadas desempeñan su actividad en el centro de trabajo, no informan percepción de aislamiento al limitarse a tiempos muy concretos. Finalmente, parece que un grupo de profesoras online señalan algunos beneficios derivados de esta condición de trabajo en soledad como evitar conflictos, economía del tiempo que se dedica a la familia y adecuación a las características personales. Esta variabilidad en las respuestas pone de manifiesto la dimensión subjetiva de los riesgos psicosociales.

Por otra parte, las participantes en sus discursos refieren distintas situaciones de conflicto persona-rol, en las que se producen demandas que son incompatibles con las creencias, valores o tareas de las docentes. $\mathrm{La}$ mayor parte de las demandas se focalizan en situaciones de evaluación, bien sean trabajos o exámenes. En este sentido, una de las situaciones que emerge de manera constante es la participación en tribunales de evaluación de los trabajos de fin de título. Formar parte de estos tribunales parece someter a las profesoras online a varias exigencias que dificultan la realización de su trabajo. Por un lado, los alumnos exigen superar obligatoriamente la evaluación, demanda que resulta incongruente con la valoración negativa de muchos de los proyectos presentados por no alcanzar los estándares de calidad. Por otro, estos alumnos cuentan con la autorización previa de su director de proyecto, que es un compañero de trabajo:

"¿Qué ocurre si, de repente, el alumno que está haciendo un trabajo que está fatal, pero que tiene autorización del director? ¿A quién acusamos, al alumno o al director? Es un dilema tremendo" (María, 10.2-67:14).

Esta situación plantea una incompatibilidad entre las demandas de los alumnos y los valores y creencias de las profesoras acerca del nivel educativo exigible. Además, refrendar la valoración positiva otorgada a estos proyectos por los directores y compañeros resulta también incongruente con estas creencias personales. Ambas condiciones parecen comportar una importante sobrecarga emocional, generando malestar en las docentes, que no saben cómo gestionarlo.

Junto a lo anterior, otro de los conflictos persona-rol que aparecen en las narraciones de las entrevistas se genera en la impartición de las asignaturas ordinarias. Frecuentemente, las demandas parecen provenir de los alumnos con referencia a las calificaciones, solicitando la superación del examen, otra prueba adicional o la flexibilización de las normas y criterios de evaluación, alcanzando en algunos casos altas cotas de intensidad. Estos conflictos con los alumnos parecen tener el mismo hilo conductor, lo que ha favorecido la creación y uso de una serie de estrategias para prevenirlos y en su caso, afrontarlos con bastante eficacia. Estas estrategias se centran en la rigurosa delimitación de los límites, normas y pautas de la dinámica de la asignatura en todos sus aspectos didácticos, y la correspondiente comunicación a los alumnos al inicio de las materias. De esta manera, se evitan las lagunas que pudieran dar lugar a distintas interpretaciones o demandas:

"Tenemos unas pautas bien transparentes de cara a los alumnos y cuando hay un problema hacemos un poco frente común ante el alumnado" (Clara, 7.2-62:10).

Por último, respecto de la relación con los alumnos, esta parece estar determinada por la dinámica y estructura de la modalidad online. Como se ha señalado, esta fórmula se caracteriza por una planificación minuciosa de todo el proceso didáctico, así como por la regulación normativa estricta de las interacciones que tienen lugar en este contexto. Esta forma de control, en ciertas organizaciones, es más flexible, lo que facilita una relación más estrecha con el alumnado. En función del grado de flexibilidad, se aprecian dos tendencias en los discursos de las profesoras online. En primer lugar, se encuentran las participantes que definen la interacción con sus alumnos como una relación marcada por el escaso contacto cara a cara y/o por escrito, que solo se permite en momentos puntuales y es de corta duración, como ocurre en las 
direcciones de trabajos de fin de título, foros y revisiones de exámenes. La falta de contacto frecuente parece poner distancia entre los alumnos y profesoras, reduciéndose la interacción a temas estrictamente académicos:

"Lo que pasa que como profesores tampoco tenemos mucha comunicación con los alumnos, o sea, es, pues eso, en el momento de la clase y luego en el foro de preguntas del profesor, entonces tampoco. La relación con los alumnos es, bueno, pues como eso, como muy profesional, en el sentido de muy académica" (Rosa, 6.2-60-3).

La segunda de las tendencias que emergen de las narraciones de las docentes parece poner de manifiesto una interacción más frecuente $\mathrm{y}$ estrecha con los alumnos, propiciada por la mayor flexibilidad organizacional. En este sentido las participantes refieren la posibilidad de contactar con sus alumnos por teléfono, realizando un seguimiento más exhaustivo, mimándolos, según sus palabras, motivándoles y guiándoles para que se produzca un aprendizaje significativo. Concretamente, sobresale la conducta y el compromiso de una de las profesoras online más allá de lo requerido en su rol laboral, que mantiene contacto telefónico, vía Skype o WhatsApp con sus alumnos e incluso plantea actividades presenciales como elemento de conexión con y entre ellos, conformándose una verdadera relación, según sus propias palabras:

"Tengo un trato muy personal con cada uno de los alumnos, entonces, incluso los alumnos acaban mucho más allá de la plataforma de teleformación, muchas veces o hablando por teléfono o haciendo Skype, es decir, tengo una relación" (Daniela, 2.1-91:1).

\section{Discusión}

Los resultados de las entrevistas muestran que uno de los principales factores de riesgo psicosocial relacionados con el puesto de trabajo son los períodos intensos de trabajo o picos de trabajo, que generan una sobrecarga mental cuantitativa. En esos momentos, ligados a la dinámica temporal de la enseñanza online y de la docencia en general, el volumen de trabajo se incrementa vertiginosamente, incluso hasta duplicar la carga de trabajo a realizar sin que se produzca un aumento del tiempo necesario para afrontarla. Esta sobrecarga cuantitativa afecta la motivación de las participantes y dificulta la conciliación familiar, especialmente, en el caso de docentes con niños en edad escolar, para las que estos picos conllevan una sobrecarga de rol derivada de las exigencias de ambas esferas, por lo que se ven obligadas a extender sus horarios de trabajo por la noche o los fines de semana. En este sentido, Moreno-Jiménez y Garrosa (2013) señalan que los cambios que se están produciendo en las organizaciones, como el incremento de la flexibilidad de los tiempos de trabajo de acuerdo con las demandas productivas y el aumento de las jornadas para cumplir con los plazos, dificultan la conciliación entre la esfera familiar y laboral. A lo anterior se añade la sobrecarga cuantitativa que experimentan las profesoras online por el desempeño de otras tareas de gestión e investigación, lo cual comporta largas jornadas de trabajo, de más de 12 horas en algunos casos, para poder cumplir con todas las exigencias. Estos hallazgos coinciden con el trabajo de Cifre y Llorens (2001), que pone de relieve que el principal factor estresante de los profesores ayudantes universitarios es la sobrecarga cuantitativa derivada de la cantidad de publicaciones que deben realizar para desarrollar su carrera universitaria.

Un segundo factor psicosocial de riesgo percibido está asociado con la subcarga mental cualitativa derivada de la rutina y monotonía de la corrección de actividades académicas, resultados que coinciden con los de Cifre, Salanova y Franco (2011).

Respecto de los factores psicosociales relacionados con el área sociolaboral, el principal aspecto percibido es el aislamiento entre las profesoras online que trabajan en su domicilio. La principal carencia se produce en la dimensión social del aislamiento (Marshall 
et al., 2007); se informa la falta de contacto con sus compañeros, la ausencia de charlas espontáneas y de apoyo emocional, es decir, la pérdida de la interacción informal en el ámbito laboral. Asociado a esto, se evidencia, haciendo alusión a la dimensión organizacional del aislamiento (Marshall et al., 2007), la necesidad de compartir dudas, de trabajar conjuntamente en un proyecto educativo común o de homogeneizar criterios en las mismas asignaturas que son impartidas por distintos profesores. Esta sensación de estar aislado se observa también en la participante que no trabaja en su domicilio. No obstante, en su caso, hace referencia más a la falta de interacción con los alumnos que con sus compañeros, puesto que compagina su trabajo en la universidad con otro trabajo presencial, estando cubierta esta necesidad social. De acuerdo con los informes de Eurofound e ILO (2017), el aislamiento y la carencia de comunicación informal es uno de los principales factores de riesgo del teletrabajo, lo que coincide con los resultados de esta investigación. En la misma línea, Cooper y Kurland (2002) subrayan que la sensación de estar aislado profesionalmente es consustancial a la modalidad del teletrabajo.

Un último factor que emerge de las narraciones de las profesoras online, aunque con menor peso, es la sobrecarga emocional derivada de los conflictos con alumnos, generalmente relativos a la evaluación. Como estos conflictos parecen tener el mismo hilo conductor, las docentes han creado y utilizado una serie de estrategias para prevenirlos $y$, en su caso, afrontarlos con bastante eficacia. Estos mecanismos se centran en la rigurosa delimitación de las normas y pautas de la dinámica de la asignatura, en todos sus aspectos didácticos y su correspondiente comunicación a los alumnos al inicio de las materias.

En síntesis, si bien el teletrabajo se ha articulado originalmente como una fórmula que promueve la autonomía y la conciliación a causa de su flexibilidad temporal y espacial, y el ajuste a las necesidades personales y fami- liares, esta investigación pone de manifiesto la existencia de distintos factores psicosociales, íntimamente ligados a esta modalidad laboral, que pueden propiciar la aparición de los llamados riesgos nuevos y emergentes (Agencia Europea para la Seguridad y Salud en el Trabajo, 2016; Brocal, 2016). En este sentido, se destaca el tecnoestrés, el burnout y las dificultades para conciliar la vida profesional y familiar como algunos de los principales riesgos psicosociales asociados al teletrabajo y, en concreto, a la profesión de profesora online (Baraza et al., 2015; Brocal, 2016; Cuervo et al., 2018; Dias y Costa, 2008; Salanova, 2007).

Por otro lado, se desprende la necesidad de implementar en los programas preventivos, que ya existen en los centros universitarios objeto de este estudio, medidas que incidan sobre los factores de riesgo en el ámbito organizacional y personal y que se ajusten a las características particulares de la mujer. A nivel de la organización, realizar ajustes realistas de la carga laboral; combinar el trabajo a domicilio con períodos de trabajo presencial, que faciliten el contacto social; regular planes formativos sistemáticos institucionalizados, en los que se actualicen no solo conocimientos relacionados con la especialidad, sino también destrezas docentes y tecnológicas (Tejada y Pozos, 2018); adecuar los salarios y proporcionar recursos laborales, como el capital psicológico (Avey, Luthans y Palmer, 2010), que motiven a las trabajadoras; $y$, a nivel personal, potenciar ciertos aspectos, como el trabajo en competencias emocionales y la promoción de hábitos saludables (Mikulic, Crespi y Radusky, 2015). Las conclusiones de este trabajo conducen a la necesidad de emprender futuras investigaciones centradas en el estudio e implementación de organizaciones saludables (aquellas que desarrollan prácticas saludables de estructurar y gestionar los procesos de trabajo), cuyo propósito no es solo intervenir sobre los aspectos disfuncionales, sino lograr la salud y bienestar de sus trabajadores, potenciando al máximo el desa- 
rrollo de la organización y de sus empleados (Salanova, Llorens y Martínez, 2016).

Finalmente, en cuanto a las limitaciones de este estudio, cabe destacar, por un lado, el tipo de metodología y el tamaño de la muestra utilizado, lo cual dificulta el proceso de generalización de los resultados y requiere la realización de estudios complementarios con muestras más amplias y metodologías de naturaleza cuantitativa; por otra parte, el hecho de garantizar el anonimato ha obligado a no indicar el lugar de procedencia de las participantes, lo que limita la comparación intermuestral. Asimismo, las edades de las teleformadoras hacen pensar en que no sean nativas digitales. Habría sido interesante, y así se recomienda para futuras investigaciones, contar con participantes nativas digitales, porque este puede ser un factor que influya en la percepción, no solo de los factores tecnológicos, sino también de los aspectos psicosociales.

\section{Referencias bibliográficas}

Agencia Europea para la Seguridad y Salud en el Trabajo (2016). Gestionar la salud y la seguridad en el trabajo. Segunda encuesta de empresas sobre riesgos nuevos y emergentes (ESE-NER-2). Recuperado de https://osha. europa.eu/es/tools-and-publications/publications/reports/scoping-study-for-a-foresight-

on-new-and-emerging-osh-risks-and-challenges/view

Alonso, M.B. y Cifre, E. (2002). Teletrabajo y salud: un nuevo reto para la psicología. Papeles Del Psicólogo, 83, 55-61.

Artazcoz, L. (2014). Aspectos metodológicos en la investigación sobre trabajo, género y salud. AREAS: Revista Internacional de Ciencias Sociales, 33, 139-153. Recuperado de https://digitum.um.es/digitum/bitstream/10201/43109/1/aspectos\%20metodologicos.pdf

Avey, J.B., Luthans, F. y Palmer, N.F. (2010). Impact of positive psychological capital on employee well-beingover time. Journal of Occupational Health Psychology, 15(1),
17-28. http://doi.org/10.1037/a0016998

Bakker, A.B., Rodriguez-Muñoz, A. y Derks, D. (2012). La emergencia de la psicología de la salud ocupacional positiva. Psicothema, 24(1), 66-72.

Baraza, X., Rimbau, E., Dalmau, I. y Llacuna, J (2015). Caso de estudio: teletrabajo y riesgos psicosociales aplicados a una universidad online. Fundación Internacional ORP. V International Conference on Occupational Risk Prevention, Santiago de Chile.

Brocal, F. (2016). Incertidumbres y retos ante los riesgos laborales nuevos y emergentes. Archivos de Prevención de Riesgos Laborales, 19, 6-9.

Cabero, J. (2006). Bases pedagógicas del e-learning. Revista de Universidad y Sociedad del Conocimiento, 3(1). Recuperado de http:// www.redalyc.org/articulo.oa?id=78030102

Castaño, C. (2010). Género y TIC. Presencia, posición y políticas. Volumen 3 de Sociedad Red. Barcelona: UOC.

Cifre, E. y Llorens, S. (2001). Burnout en profesores de la UJI: un estudio diferencial. Forum de recerca, 7, 2-11.

Cifre, E., Salanova, M. y Franco, J. (2011). Riesgos psicosociales de hombres y mujeres en el teletrabajo ¿una cuestión de diferencias? Gestión práctica de riesgos laborales, 82, 29-36.

Cooper, C.D. y Kurland, N.B. (2002). Telecommuting, professional isolation, and employee development in public and private organizations. Journal of Organizational Behavior, 532, 511-532. http://doi.org/10.1002/job.145

Crawford, J.O., Maccalman, L. y Jackson, C.A. (2011). The health and well-being of remote and mobile workers. Occupational Medicine, 61, 385-394. http://doi.org/10.1093/occmed/ kqr071

Cuervo, T., Orviz, N., Arce, S. y Fernández-Suárez, I. (2018). Tecnoestrés en la Sociedad de la Tecnología y la Comunicación: revisión Bibliográfica a partir de la Web of Science. Archivos de Prevención de Riesgos Laborales, 21(1), 18-25. http://doi.org/10.12961/ aprl.2018.21.01.4

Dias, M. y Costa, J. (2008). Impacto psicosocial 
de la Tecnología de la Información y Comunicación (TIC): estrés, daños físicos y satisfacción laboral. Acta Colombiana de Psicología, 11(2), 127-139.

Domínguez, J.A. (2004). Riesgo Psicosocial en la universidad: estresores propios del docente universitario. Revista Digital de Prevención, 2, $1-38$.

Eurofound and the International Labour Office, ILO (2017).Working anytime, anywhere: The effects on the world of work. Publications Office of the European Union, Luxembourg and the International Labour Office, Geneva.

European Trade Union Committee for Education (2011). Evaluation of the impact of psychosocial hazards on teachers 2010-2012. Recuperado de https://www.csee-etuce.org/en/ policy-issues/teachers-health-and-safety/ work-related-stress/876-project-stress-iii

Gálvez, A.M. (2008). Conciliación de la vida laboral y familiar en mujeres que trabajan con nuevas tecnologías de la comunicación y la comunicación: un análisis psicosocial $y$ cultural de las estrategias desplegadas. Ministerio de Igualdad: Instituto de la Mujer. Recuperado de http://www.inmujer.gob.es/ areasTematicas/estudios/estudioslinea2009/ docs/775Concvidalaboral.pdf

Lawshe, C.H. (1975). A quantitative approach to content validity. Personnel Psychology, 28, 563-575. http://dx.doi. org/10.1111/j.1744-6570.1975.tb01393.x

Lichtman, M. (2013). Qualitative research for the social sciences. California: Sage Publications.

Marshall, G.W., Michaels, C.E. y Mulki, J.P. (2007). Workplace isolation: Exploring the construct and its measurement. Psychology \& Marketing, 24(3), 195-223. https://doi. org/10.1002/mar.20158

Merino, J.M., Meneses, E.L. y Ballesteros-Regaña, C. (2008). El profesor universitario en la Sociedad de la Información y la Comunicación. Espacio y Tiempo: Revista de Ciencias Humanas, 22, 213-231.

Mikulic, I.M., Crespi, M. y Radusky, P. (2015). Construcción y validación del inventario de competencias socioemocionales para adultos
(ICSE). Interdisciplinaria, 32(2), 307-329. https://doi.org/10.16888/interd.2015.32.2.7

Moreno-Jiménez, B. y Garrosa, E. (2013). Salud Laboral. Factores y riesgos psicosociales. En B. Moreno-Jiménez y E. Garrosa (Eds.), Salud laboral. Riesgos psicosociales y bienestar laboral (pp. 419-435). Madrid: Pirámide.

Organización Internacional del Trabajo (OIT, 2011). Manual de buenas prácticas en teletrabajo. Recuperado de http://www.ilo.org/ wcmsp5/groups/public/---americas/---ro-lima/---ilo-buenos_aires/documents/publication/wcms_bai_pub_143.pdf

Pérez, C. y Gálvez, A.M. (2009). Teletrabajo y vida cotidiana: ventajas y dificultades para la conciliación de la vida. Athenea Digital, 15, 57-79. https://doi.org/10.5565/rev/athenead/ v0n15.597

Pulido, N., Puentes, A., Luney, Z., López, D.R. y García, M. (2015). Análisis bibliométrico de la producción científica sobre riesgo psicosocial laboral, publicada entre 2000 y 2010. Diversitas, 11, 147-161.

Querol, V. y Sáez, E. (Eds.). (2011). Ideas, tendencias y nuevas formas de trabajo para la economía del conocimiento. Servicio de Comunicación y Publicaciones de la Universidad Jaume I: Castellón.

Salanova, M. (2007). Nuevas tecnologías y nuevos riesgos psicosociales en el trabajo. Revista Digital de Salud y Seguridad en el Trabajo, 1, $1-21$.

Salanova, M., Llorens, S. y Martínez, I.M. (2016). Aportaciones desde la psicología organizacional positiva para desarrollar organizaciones saludables y resistentes. Papeles del Psicólogo, 37(3), 177-184. Recuperado de http:// www.papelesdelpsicologo.es/pdf/2773.pdf

Sebastián, O. y Del Hoyo, M.A. (2002). La carga mental de trabajo. Madrid: INSHT.

Seidman, I. (2006). Interviewing as qualitative research: A guide for researchers in education and the social sciences. New York: Teachers College, Columbia University.

Tejada, F. y Pozos, K.V. (2018). Nuevos escenarios y competencias digitales docentes: hacia la profesionalización docente con TIC. Profe- 
sorado. Revista de Currículum y Formación del Profesorado, 22(1), 25-51.

Ulin, P.R., Robinson, E.T. y Tolley, E.E. (2006). Investigación aplicada en salud pública. Métodos cualitativos. Washington, D.C: Organización Panamericana de la Salud. Recuperado de http://iris.paho.org/xmlui/bitstream/ handle/123456789/729/9275316147.pdf
Valenzuela, M.E. y Mora, C. (2009). Trabajo doméstico: un largo camino hacia el trabajo decente. Santiago de Chile: Organización Internacional del Trabajo.

Velásquez, C.M. y Vera, M. (2018). Teletrabajo: una revisión teórica sobre sus ventajas $\mathrm{y}$ desventajas. INVESTIGATIO, 10, 41-53. https://doi.org/10.31095/irr.v0i10.194

Recibido: 18 de mayo de 2018

Aceptado: 22 de abril de 2020 